\title{
Expanding the Distinctive Neuroimaging Phenotype of ACTA2 Mutations
}

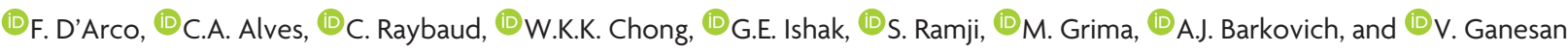

\begin{abstract}
BACKGROUND AND PURPOSE: Arg179His mutations in ACTA2 are associated with a distinctive neurovascular phenotype characterized by a straight course of intracranial arteries, absent basal Moyamoya collaterals, dilation of the proximal internal carotid arteries, and occlusive disease of the terminal internal carotid arteries. We now add to the distinctive neuroimaging features in these patients by describing their unique constellation of brain malformative findings that could flag the diagnosis in cases in which targeted cerebrovascular imaging has not been performed.
\end{abstract}

MATERIALS AND METHODS: Neuroimaging studies from 13 patients with heterozygous Arg179His mutations in ACTA2 and 1 patient with pathognomonic clinicoradiologic findings for ACTA2 mutation were retrospectively reviewed. The presence and localization of brain malformations and other abnormal brain MR imaging findings are reported.

RESULTS: Characteristics bending and hypoplasia of the anterior corpus callosum, apparent absence of the anterior gyrus cinguli, and radial frontal gyration were present in $100 \%$ of the patients; flattening of the pons on the midline and multiple indentations in the lateral surface of the pons were demonstrated in $93 \%$ of the patients; and apparent "squeezing" of the cerebral peduncles in $85 \%$ of the patients.

CONCLUSIONS: Because $\alpha$-actin is not expressed in the brain parenchyma, only in vascular tissue, we speculate that rather than a true malformative process, these findings represent a deformation of the brain during development related to the mechanical interaction with rigid arteries during the embryogenesis.

$\mathbf{T}$ he cerebral arteriopathy associated with Arg179His mutations in ACTA2 is a prototypical example of nonatherosclerotic cerebral arteriopathies, some of which are Mendelian disorders. ${ }^{1}$ Patients

Received June 13, 2018; accepted after revision August 16.

From the Departments of Radiology (F.D'A., W.K.K.C.) and Neurology (V.G.), Great Ormond Street Hospital for Children National Health Service Foundation Trust, London, UK; Radiology Department (C.A.A.), Hospital Das Clinicas, Sao Paulo, Brazil; Department of Diagnostic Imaging (C.R.), Hospital for Sick Children, Toronto, Ontario, Canada; Department of Radiology (G.E.I.), Seattle Children's Hospital, University of Washington School of Medicine, Seattle, Washington; Department of Radiology (S.R.), Imperial College Healthcare National Health Service Trust, London, UK; Department of Radiology (M.G.), University Hospital of North Staffordshire National Health Service Trust, Stoke-on-Trent, UK; Department of Radiology and Diagnostic Imaging (A.J.B.), University of California, San Francisco, San Francisco, California; and Neuroscience Unit (V.G.), UCL Great Ormond Street Institute of Child Health, London, UK.

Felice D'Arco and Cesar A. Alves contributed equally to the article.

This project was supported by the National Institute for Health Research Biomedical Research Centre at Great Ormond Street Hospital for Children National Health Service Foundation Trust and University College London.

Please address correspondence to Felice D'Arco, MD, Great Ormond St Hospital for Children NHS Trust, Department of Radiology, London WCIN 3JH, UK; e-mail: darcofel@gmail.com; @FeliceDArco

-- Indicates open access to non-subscribers at www.ajnr.org

三 Indicates article with supplemental on-line table.

http://dx.doi.org/10.3174/ajnr.A5823 with the ACTA2 mutation have distinctive clinical (multisystem smooth-muscle involvement) and angiographic features ${ }^{2}$ specifically, a combination of ectasia and stenosis, a straight arterial course, absence of basal collaterals, and more widespread cerebrovascular involvement in comparison with Moyamoya disease. ${ }^{2}$ The diagnosis is suggested by these imaging features and has important implications for the management of the patient (increased risks associated with arterial instrumentation) and other family members, but it also provides important mechanistic insights that may be more generalizable. ${ }^{1}$ Previously, the imaging phenotype associated with ACTA2 mutations had been confined to cerebrovascular abnormalities and associated leukoencephalopathy, ${ }^{3}$ apart from a single case report of a patient with a dysmorphic corpus callosum. ${ }^{4}$

Here we expand the neuroimaging phenotype and describe characteristic brain parenchymal abnormalities that could flag the diagnosis when targeted cerebrovascular imaging has not been performed.

\section{MATERIALS AND METHODS}

We reviewed in detail the brain MR imaging findings in a cohort of patients with ACTA2 mutations to describe the characteristics of brain congenital abnormalities in addition to known neuroan- 


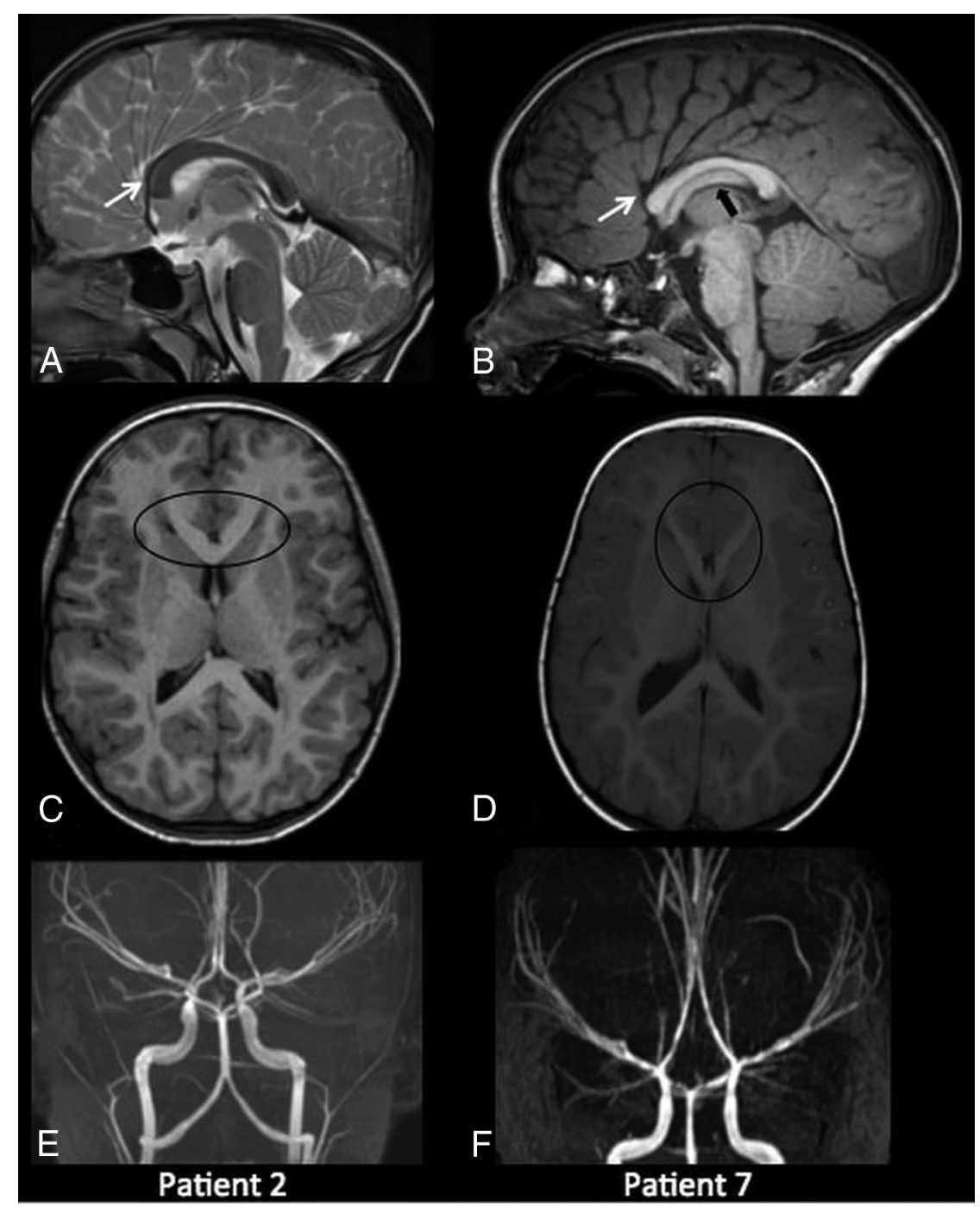

FIG 1. Supratentorial abnormalities in patients with the ACTA2 mutation. Upper row: sagittal T2-weighted image $(A)$ and sagittal TTWI $(B)$ show typical hypoplasia and bending of the anterior corpus callosum with associated abnormal radial gyration of the frontal lobes and a deficient anterior cingulate gyrus (white arrows). In patient 7, there is a horizontal orientation of the fornix (black arrow in B), which is also markedly thick. Middle row: axial TIWI ( $C$ and $D$ ) demonstrates characteristic V-shaped anterior corpus callosum (black circle). Lower row: MR angiography maximum-intensity-projection anteroposterior view $(E$ and $F$ ) shows typical neurovascular abnormalities in patients with ACTA2 mutation.
Another patient had no genetic confirmation (lost at follow-up) but showed typical radiologic and clinical phenotypes that are considered pathognomonic for ACTA2 mutation $^{2,5}$ and thus was included in the study.

Images were reviewed for quality and co-reported by 2 pediatric neuroradiologists (F.D'A. and W.K.K.C.), who reported the presence and localization of brain malformations (ie, the presence of cortical malformations; abnormal shape of the brain stem; abnormal relative size of the midbrain, pons, and medulla oblongata; abnormal shape and size of the corpus callosum; and abnormal gyration) (On-line Table). MR angiography or digital subtraction angiography or both, when available, were also reviewed for typical ACTA2-related cerebrovascular anomalies as described in literature. ${ }^{2}$

The presence of associated ischemic brain damage was also reported and divided into large territorial infarction of the brain and evidence of watershed infarctions/small vessel disease.

The presence of specific brain malformations found in our cohort was compared with known similar brain malformation patterns and analyzed in view of known embryologic knowledge ${ }^{6}$ to elucidate possible pathogenetic mechanisms.

Review through the electronic data management systems of the MR imaging scans and clinical data of all the patients from the main institution (ie, Great Ormond Street Hospital for Children) with a diagnosis of other forms of neurovascular dysplasia (eg, Moyamoya disease) excluded the presence of similar brain abnormalities. giographic findings previously described. ${ }^{2}$ Clinically acquired, anonymized brain and cerebrovascular imaging studies from 13 unrelated patients with heterozygous Arg179His mutations in ACTA2 were retrospectively included from 4 pediatric hospitals and 3 general university hospitals (University Hospital of North Midlands; Hospital Das Clinicas, Sao Paulo, Brazil; Imperial College Healthcare National Health Service Trust, London, UK), with appropriate governance permissions from each site. The patients were selected after a search for ACTA2 mutation in the electronic data management systems of the centers involved in the study (past 10 years). Inclusion criteria were confirmed mutation and/or pathognomonic neuroradiologic and clinical findings and availability of MR imaging of diagnostic quality.

This included re-analysis of 8 patients already published by $\mathrm{Mu}-$ not et al. ${ }^{2}$ All 13 patients had the genetic diagnosis confirmed in a clinically accredited laboratory as part of their clinical evaluation.

\section{RESULTS}

Clinical, radiologic, and genetic findings in our patients are summarized in the On-line Table. Thirteen patients had heterozygous missense Arg179His in ACTA2, and 1 patient without genetic confirmation had clinical and radiologic findings pathognomonic for ACTA2 mutation (subject 14) and was included in the case series.

All patients had the neuroangiographic features previously described in the ACTA2 Arg179His mutation: namely, dilation of the proximal internal carotid arteries, occlusion or narrowing of the distal internal carotid arteries, straight "broomstick-like" arteries of the circle of Willis, and absence of Moyamoya collaterals (Fig $1 E,-F)$. Multiple areas of abnormal signal in keeping with small vessel disease and sometimes frank supratentorial infarctions in a different stage of maturation were commonly observed. 


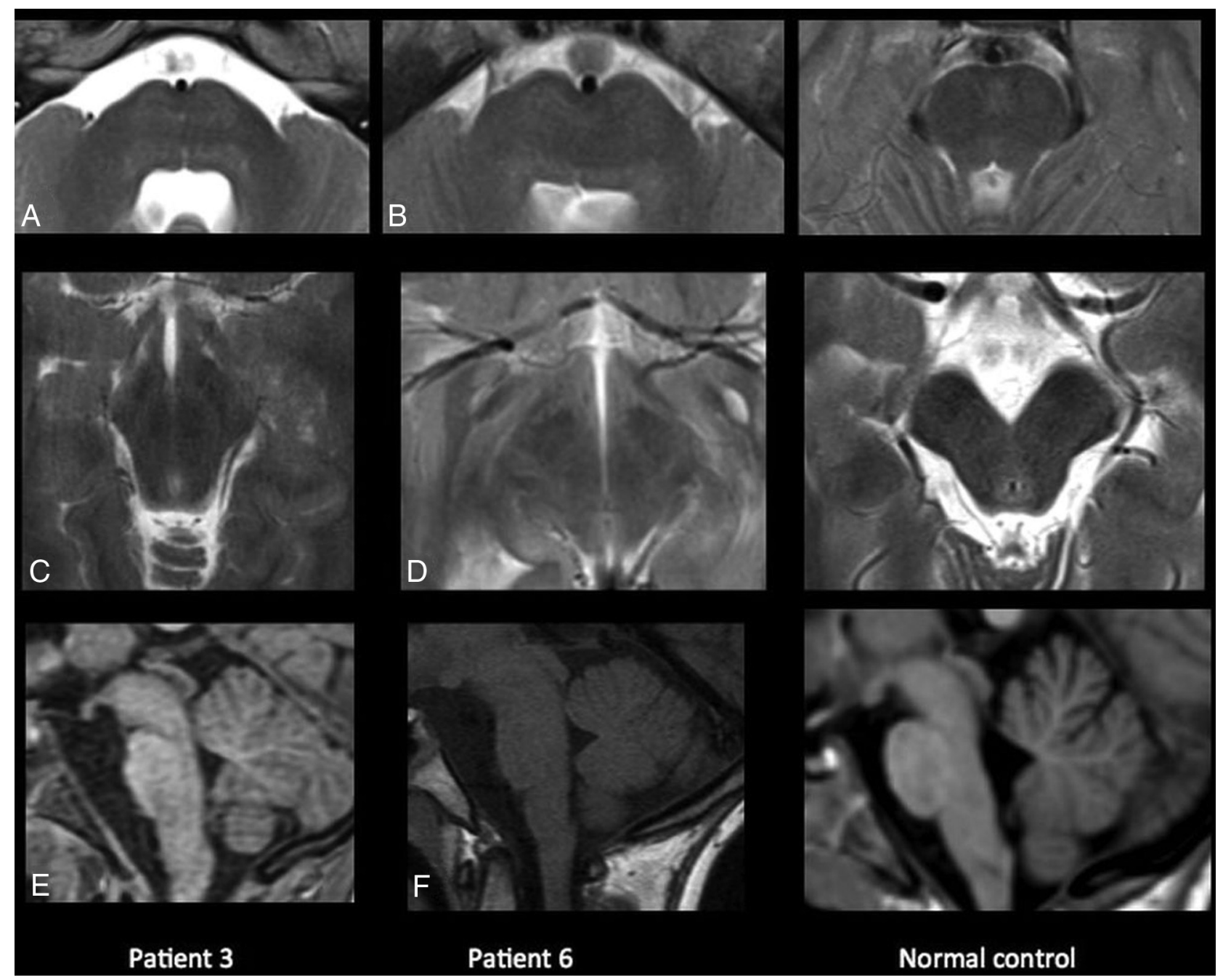

FIG 2. Infratentorial malformations in ACTA2 mutation. Upper row: axial T2WI ( $A$ and $B)$ at the level of the pons. The twin peaks sign is demonstrated; the pons is flattened with reduction of the anteroposterior diameter and an impression of the basilar artery on the anterior surface with consequent presence of 2 symmetric prominences resembling twin mountains. Middle row: axial T2WI at the level of the cerebral peduncles $(C$ and $D)$ shows mild antero-posterior elongation of the midbrain with reduction of the laterolateral diameter and squeezing of the cerebral peduncles. Right column: normal for comparison.

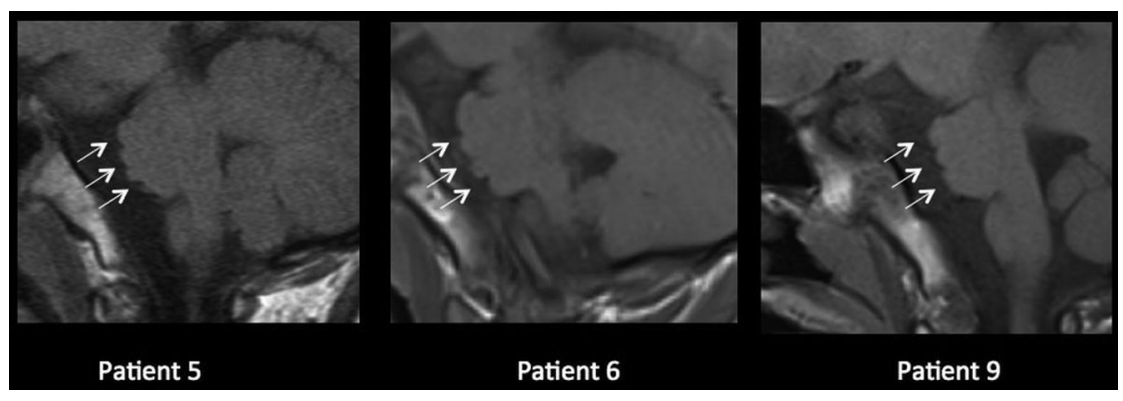

FIG 3. In the parasagittal slice, the patients show multiple indentations on the surface of the pons. We speculate that these may be due to stretching of the straightened pontine arterial branches.

All patients showed different degrees of abnormal brain morphology, namely the following:

1) Bending (excessive curvature inferiorly and anteriorly) and hypoplasia (rostrum not well-formed and flattened genu) of the anterior corpus callosum (Fig 1A,-B) with a relatively normal or mildly hypoplastic posterior corpus callosum were present. On axial images, the anterior corpus callosum demonstrates a characteristic V-shaped appearance (Fig $1 C,-D)$. This finding was present in all (100\%) patients.

2) Abnormal radial gyration of the frontal lobes and a deficient anterior cingulate gyrus (Fig 1A, -B) (100\%) was present.

3) “Twin peaks" pons (appreciable in all the patients with the exception of patient 1: 93\%): The pons was flattened with subjective reduction of the anteroposterior diameter noted on the midline and an impression of the basilar artery on the anterior surface with the consequent presence of 2 symmetric prominences resembling twin mountains (Fig 2). In the parasagittal slice, the patients had multiple indentations on the surface of the pons (Fig 3), probably due to the straightened pontine arterial branches creating compression of the pontine surface (see the "Discussion"). 


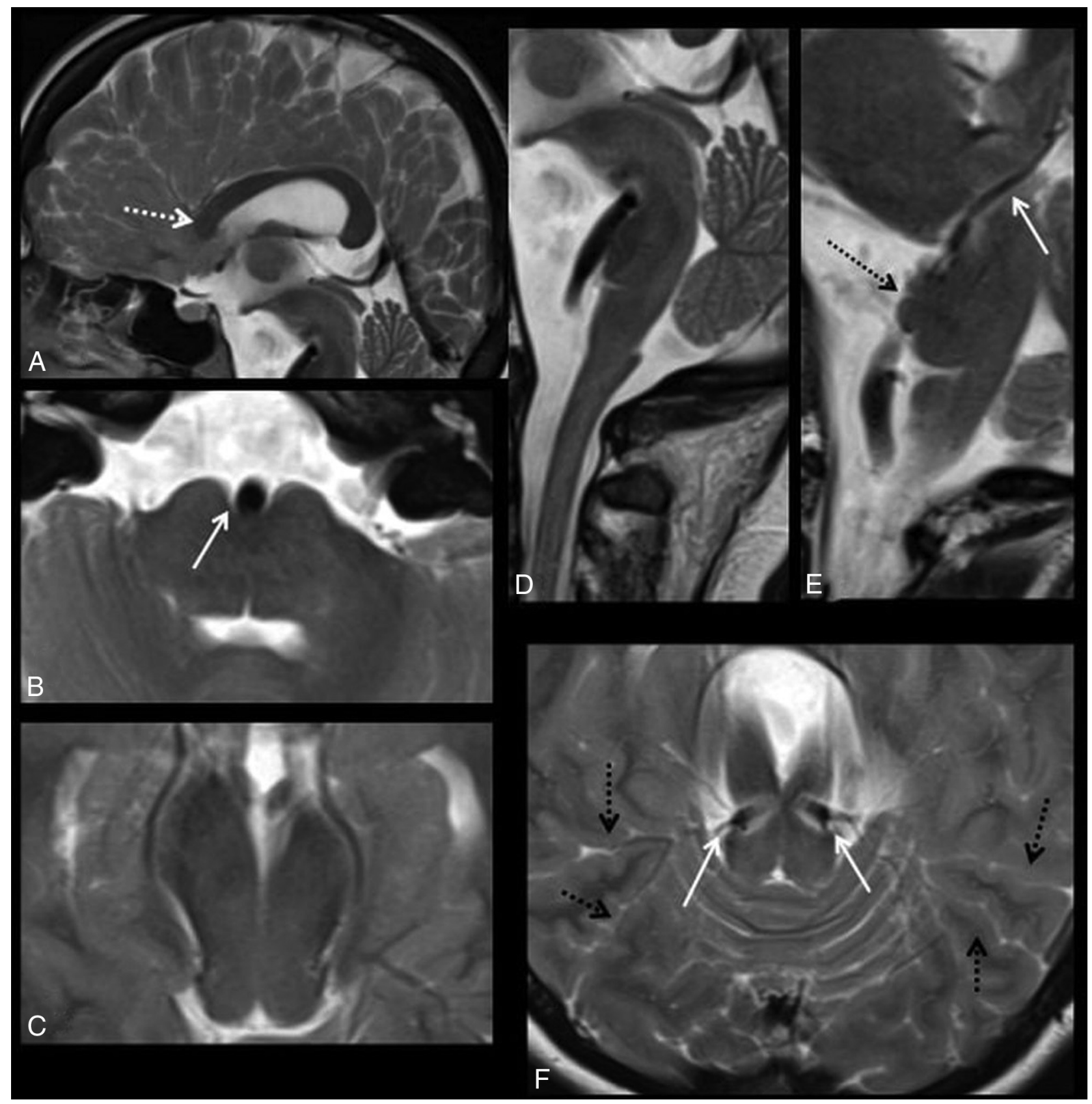

FIG 4. Neuroradiologic findings in an adult patient without a confirmed ACTA2 mutation (patient 15). Sagittal T2WI (A) and axial T2WI at the level of the pons $(B)$ and the midbrain $(C)$ show marked callosal anterior bending (dotted arrow in $A$ ), the twin peaks pontine sign (arrow in $B$ ), and reduction of the laterolateral diameter of the midbrain with a squeezed cerebral peduncle. $D$, Sagittal T2WI demonstrates a marked basilar impression on the pons and anterior bending of the midbrain. The parasagittal right slice $(E)$ demonstrates indentation of the lateral surface of the pons (dotted black arrow) and a straight course of the posterior cerebral arteries (white arrow). Axial T2WI (F) at the level of the proximal segment of the posterior cerebral arteries (pontomesencephalic junction) shows marked compression of the brain stem at that level related to straightening of the arteries (white arrows) and radial gyration of the posterior temporal lobes (black dotted arrows).

4) Apparent "squeezing" of the cerebral peduncles in the midbrain (Fig $2 C,-D)$ was present in 12 patients $(85 \%)$.

5) A variable degree of horizontal orientation and thickening of the fornix (ie, fornix parallel to the corpus callosum) was present in all patients (Fig $1 A,-B$ ).

The radiologic and clinical findings of the patient without confirmed mutations were similar to those in the confirmed cases, with the typical ACTA2 cerebrovascular changes. ${ }^{2}$

Most interesting, we also found an adult patient with a similar and pronounced ACTA2-like cerebrovascular phenotype (patient
15 in the On-line Table), which showed the same spectrum of brain abnormalities, straightening of posterior circulation arteries, and abnormal radial gyration also involving the posterior temporal lobes (Fig 4). This patient refused genetic testing and did not have pathognomonic clinical findings associated with ACTA2 mutation. Nevertheless, this case is extremely interesting for understanding the possible pathophysiology of the brain abnormalities related to vascular dysplasia (see the "Discussion").

In 8 subjects (patients 1, 5, 8, 10,11,12, 13, and 15), follow-up MR imaging was not available. The others had a different evolu- 
tion of the ischemic cerebral lesions. In all patients with follow-up available, the abnormalities in the corpus callosum, gyration, and brain stem were stable with time as well as the degree of arteriopathy. None of the patients showed cortical malformations such as focal cortical dysplasia, polymicrogyria, agyria, or pachygyria, as described in literature. ${ }^{7}$

\section{DISCUSSION}

Actin is an abundant protein in eukaryotic organisms and plays an essential role in the protein-protein interactions. The actin protein represents a monomeric subunit of 2 main varieties of filaments in cells that make up the cytoskeleton and form part of the contractile apparatus in muscle cells. The mammalian genome comprises 6 distinct actin isoforms ( $\alpha$-skeletal, $\alpha$-cardiac, $\alpha$-smooth, $\beta$-cytoplasmic, $\gamma$-smooth, and $\gamma$-cytoplasmic actin) encoded by 6 different genes. ${ }^{8}$ Alpha-smooth-muscle actin, encoded by ACTA2, located on 10q22-q $24,{ }^{9}$ is a principal element of the contractile units of vascular smooth-muscle cells but is not expressed in the brain parenchyma. However, $\alpha$-actin cross-reacts with $\gamma$-actin to reinforce the cytoskeleton. ${ }^{10}$ The arterial phenotype in Arg179His ACTA2 mutations, with ectasia of the large arteries and occlusive disease in the small arteries, appears to reflect the local influence on the presence or absence of elastin within the arterial wall on the vascular phenotype. ${ }^{2}$ These arterial features are also observed in the mouse model. ${ }^{11}$ Vascular smooth-muscle cells are derived from the mesoderm in the posterior fossa/brain stem/thalami and form the neural crest supratentorially (anterior neural plate) ${ }^{12}$; however, there are no data, to the best of our knowledge, to suggest differences in expression of ACTA2 related to different embryologic origins.

The occlusive disease observed in intracranial vessels is a result of fibrosis, thickening of the vascular wall, flattening and disorganization of the internal lamina, and proliferation of smooth-muscle cells. ${ }^{13}$ We previously postulated that this process renders the arteries more rigid and less deformable. ${ }^{2}$ Arterial growth follows the contours of brain growth and gyration during normal development. $^{12}$ Thus, we speculate that in patients with these ACTA2 mutations, the increased rigidity of the intracranial arteries results in both the characteristic "straight" appearance and the morphologic brain changes that we have described as a consequence of the local mechanical effect from these vessels. Interestingly, the muscular layers do not appear in the basal perforator vessels until the gestational age of 27 weeks and, progressively, until term over the convexity. However studies on rat embryos show that actin expression in the vasculature starts very early, ${ }^{14}$ and histologic specimens in subjects with ACTA2 mutations demonstrate that the rigidity is also due to abnormality in elastic and intima laminae. ${ }^{13}$ Furthermore, although the shape of the corpus callosum is complete by the gestational age of 20 weeks, this structure enlarges together with the connectivity and the development of the cortex ${ }^{6}$; thus, it is still possible that interaction with abnormally formed vessels is responsible for the observed deformity despite these differences in the embryologic age of development. In addition, because the actin cytoskeleton participates directly or indirectly in almost every aspect of neuronal development and function, ${ }^{15}$ any instability in the cytoskeleton resulting from abnormal cross-linkage between actin subtypes could also influence neuronal migration. ${ }^{8}$ Alpha-actin is not expressed in the brain parenchyma but only in vessels (different from other isoforms), so it is unlikely that a mutation of this isoform will directly influence brain development, but it is possible that cross-regulation between different isoforms may play a role in subjects with the ACTA2 mutation.

There are other examples of malformative disorders being related to local mechanical factors, for example, arachnoid cysts with surrounding brain hypoplasia and Chiari I malformation, in which a small posterior fossa results in distortion and inferior displacement of the cerebellar tonsils, which is why the term "Chiari I deformity" was proposed instead of "malformation."16

In patients with the ACTA2 mutation, the abnormal arterial morphology and structural brain abnormalities parallel each other in both supratentorial and infratentorial parenchymauniversally so in the anterior part of the corpus callosum, anterior cingulate gyrus, and abnormal radial frontal gyration, and variably in the brain stem. In fact, the characteristic twin peaks appearance of the pons in the axial plane seems to be related to impression by the basilar artery as well as the indentation of the lateral pontine surface that may be due to the impression by the pontine arteries coming from the basilar artery (Fig 3).

We have observed a particularly extreme example of this phenotype in a genetically untested patient who showed neuroradiologic features similar to those in ACTA2 mutations (patient 15). In this patient, the posterior cerebral arteries are also severely straightened and are associated with abnormal radial gyration involving the posterior temporal lobes and marked brain stem compression (Fig 4). The vascular phenotype present in this patient is similar to that in the others and is only described in patients with ACTA2 mutation, with the exception of an isolated case report ${ }^{17}$ in which a mutation was not found and brain images were very similar to those in our patient. Thus, even though it is possible that this patient (despite neurovascular findings) does not have an ACTA2 mutation, we think that the striking association between severe vascular and brain phenotypes in this subject is supportive of our hypothesis that brain abnormalities are secondary to vessel rigidity.

The apparent absence/definition of a segment of the anterior cingulate and the frontal radial pattern are both likely associated with a callosal abnormality at that level, which itself probably translates into the axial callosal V-shape. The horizontal fornix (or rather, the fornix that is parallel to the anterior callosum) results from an abnormally developed septum pellucidum (ie, too thick), which itself may well relate to the abnormal cingulate. ${ }^{6}$

The brain malformation features do not appear to have a clear clinical correlate in patients with Arg179His ACTA2 mutations; epilepsy is rare, other than in the context of brain ischemia, and intellectual outcomes again seem related to brain injury rather than to developmental abnormalities per se. However, the extreme reproducibility of the brain phenotype could represent an asset in the diagnosis when neuroangiographic studies are not available; for instance, in patient 8 , standard MR imaging sequences showed typical bending of corpus callosum and radial frontal gyration that triggered the addition of an MRA sequence, which confirmed the radiologic diagnosis.

These observations contribute to defining the distinctive neu- 
roradiologic features of ACTA2 mutations, as well to shedding light on mechanisms, both genetic and mechanical, that result in structural changes to the brain and its vasculature.

\section{CONCLUSIONS}

We describe a characteristic and potentially pathognomonic (in specific clinical context) brain phenotype in patients with ACTA2 mutations and/or the typical clinical and neurovascular picture. A possible explanation for these brain imaging findings, which can be helpful in diagnosis, is a mechanical effect on the brain parenchyma during development by abnormal rigid vessels with possible contribution of cross-regulation between different actin isoforms.

Disclosures: Marius Grima-UNRELATED: Employment: University Hospitals of North Midlands, Comments: I am a salaried doctor in this institution; Travel/Accommodations/Meeting Expenses Unrelated to Activities Listed: University Hospitals of North Midlands, Comments: study-leave budget.

\section{REFERENCES}

1. Ganesan V, Prengler M, Wade A, et al. Clinical and radiological recurrence after childhood arterial ischemic stroke. Circulation 2006;114:2170-77 Medline

2. Munot P, Saunders DE, Milewicz DM, et al. A novel distinctive cerebrovascular phenotype is associated with heterozygous Arg179 ACTA2 mutations. Brain 2012:135(Pt 8):2506-14 CrossRef Medline

3. Moosa AN, Traboulsi EI, Reid J, et al. Neonatal stroke and progressive leukoencephalopathy in a child with an ACTA2 mutation. J Child Neurol 2013;28:531-34 CrossRef Medline

4. Meuwissen ME, Lequin MH, Bindels-de Heus K, et al. ACTA2 mutation with childhood cardiovascular, autonomic and brain anomalies and severe outcome. Am J Med Genet A 2013:161A:1376-80 CrossRef Medline

5. Milewicz DM, Østergaard JR, Ala-Kokko LM, et al. De novo ACTA2 mutation causes a novel syndrome of multisystemic smooth muscle dysfunction. Am J Med Genet A 2010:152A: 2437-43 CrossRef Medline

6. Raybaud C. The corpus callosum, the other great forebrain commissures, and the septum pellucidum: anatomy, development, and malformation. Neuroradiology 2010;52:447-77 CrossRef Medline

7. Di Donato N, Chiari S, Mirzaa GM, et al. Lissencephaly: expanded imaging and clinical classification. Am J Med Genet A 2017;173: 1473-88 CrossRef Medline

8. Perrin BJ, Ervasti JM. The actin gene family: function follows isoform. Cytoskeleton (Hoboken) 2010;67:630-34 CrossRef Medline

9. Ueyama H, Bruns G, Kanda N. Assignment of the vascular smooth muscle actin gene ACTSA to human chromosome 10. Jinrui Idengaku Zasshi 1990;35:145-50 CrossRef Medline

10. Belyantseva IA, Perrin BJ, Sonnemann KJ, et al. Gamma-actin is required for cytoskeletal maintenance but not development. Proc Natl Acad Sci U S A 2009;106:9703-08 CrossRef Medline

11. Yuan SM. $\boldsymbol{\alpha}$-Smooth muscle actin and ACTA2 gene expressions in vasculopathies. Braz J Cardiovasc Surg 2015:30:644-49 CrossRef Medline

12. Raybaud C. Normal and abnormal embryology and development of the intracranial vascular system. Neurosurg Clin NAm 2010;21:399426 CrossRef Medline

13. Georgescu MM, Pinho Mda C, Richardson TE, et al. The defining pathology of the new clinical and histopathologic entity ACTA2related cerebrovascular disease. Acta Neuropathol Commun 2015; 3:81 CrossRef Medline

14. de Ruiter MC, Poelmann RE, van Iperen L, et al. The early development of the tunica media in the vascular system of rat embryos. Anat Embryol (Berl) 1990;181:341-49 Medline

15. Cheever TR, Ervasti JM. Actin isoforms in neuronal development and function. Int Rev Cell Mol Biol 2013;301:157-213 CrossRef Medline

16. Poretti A, Ashmawy R, Garzon-Muvdi T, et al. Chiari type 1 deformity in children: pathogenetic, clinical, neuroimaging, and management aspects. Neuropediatrics 2016;47:293-307 CrossRef Medline

17. Nagarajan K, Swamiappan E, Anbazhagan S, et al. "Twig-like” cerebral vessels are not pathognomonic for ACTA A2 mutations: a case report. Interv Neuroradiol 2018;24:463-68 CrossRef Medline 